\title{
L'ONTOLOGIE PHOTOGRAPHIQUE : PROCÉDÉS, GENRES ET ENJEUX
}

\author{
Catherine Saouter ${ }^{1}$
}

Le présent texte a pour objet une réflexion sur quelques repères épistémologiques proposés pour l'étude de la photographie, tenant compte de trois caractéristiques estimées essentielles afin de décrire ce mode d'expression visuelle. Premièrement, le poids historique d'un procédé technique qui détermine la photographie comme un médium lié ontologiquement à la lumière. Deuxièmement, la double nature sémiotique des énoncés photographiques qui en font à la fois des constructions référentielles et des initiatives discursives dans le corpus des représentations et des expressions culturelles. Troisièmement, la place des objets photographiques dans le champ socio-culturel, découlant de l'appropriation quasi universelle du procédé technique.

\section{De l'innovation technique au médium universel}

Dans l'esprit autant que dans la lettre, la photographie est une émanation du XIXe siècle positiviste, industriel et capitaliste.

1 Université du Québec à Montréal.

Recherches en communication, $\mathrm{n}^{\circ} 27$ (2007). 
Deux pôles, documentaire et esthétique, se dessinent très rapidement entre lesquels prend une place médiane, de façon lente mais déterminante, ce qu' on appellera plus tard le photojournalisme. Le pôle de la documentation concerne cet usage de la photographie qui comprend le nouveau médium comme un moyen automatique de capter une image parfaite et authentique de la réalité. La photographie, dans la poursuite de l'imitatio initiée à la Renaissance, rend obsolètes les performances du dessin. La photographie donne une trace directe des choses grâce à la lumière ; elle délivre des limites, lenteurs et imperfections de la main du dessinateur. Ce point de vue positiviste est défendu avec enthousiasme par des scientifiques comme François Arago (1839) ou Gay-Lussac. Par des mécènes et des commanditaires, comme le Prince Albert (et la reine Victoria) qui envoyèrent Roger Fenton photographier la guerre de Crimée. Par des éditeurs comme Lerebours qui, en publiant deux volumes d'Excursions daguerriennes en 1841 et 1842 à Paris, sur l'Italie et le Moyen-Orient, fit connaître les toutes premières vues photographiques de l'Acropole ramenées par Gustave Gaspard Joly de Lotbinière ${ }^{1}$.

Tous alimentent un débat sur les fonctions et la valeur de la photographie. Nombre d'entre eux ont été formés par les écoles des beauxarts, tous sont imprégnés d'une culture du tableau, paradigme incontesté2. En effet, dans ce débat très fortement idéologisé, dans lequel s'entremêlent la question du beau et l'apologie positiviste de la science, une prémisse sous-jacente n'est pas remise en question. Le modèle de la photographie, le paradigme de la bonne image, d'un point de vue documentaire autant qu'esthétique, est le tableau modélisé par la hiérarchie des genres ${ }^{3}$ telle qu' on l'emploie depuis plusieurs siècles. Lotbinière fait des paysages, Nadar fait des portraits. Daguerre et Fenton agencent des natures mortes devant l'objectif. Le proto-photojournalisme prend

1 E. BROWN, "Les premières images par daguerréotype au monde - le photographe canadien Pierre Gustave Gaspard Joly de Lotbinière", Ottawa, L'archiviste/ The Archivist, no 118, 1999, pp. 23-29 et C. SAOUTER, Images est sociétés - Le progrès, les médias, la guerre, Montréal, Les Presses de 1'Université de Montréal, 2003, pp. 19-20.

2 V. STOICHITA, L'instauration du tableau, Paris, Méridiens Klincksieck, 1993.

3 A. FÉLIBIEN DES AVAUX, Conferences de l'academie royale de peinture et de sculpture Pendant l'année 1667, à Paris, chez Leonard, imprimeur ordinaire du Roy, ruë S. Jacques, à l'Escu de Venise, M.DC.LXIX, avec privilège du Roy, réimpression anastatique The Printed Sources of Western Art, vol. 8, Portland, Collegium Graphicum, 1972, non paginé. 
fondamentalement appui sur la scène de genre. Ce découpage de la réalité, repris tout autant par la bande dessinée et le cinématographe à la fin du XIXe siècle, donne lieu à ce qui est appelé aujourd'hui taille des plans et conforme toujours toutes les productions jusqu'au dernier téléjournal. La pratique amateure et son poids quantitatif donnent certainement la preuve la plus évidente de la profonde intégration de cette écriture par l'image : paysage, portrait, scène de genre composent les innombrables pièces des albums de famille et, de nos jours, des blogs personnels.

Ressemblance parfaite entre l'image et ce qu'elle donne à voir, formalisation des cadrages : il ne faut jamais perdre de vue que la photographie est une solution technique qui signale une étape cruciale - perçue comme un aboutissement indépassable par les contemporains de l'invention - dans une recherche poursuivie depuis la Renaissance, l'invention de la camera obscura et de la perspective monoculaire ${ }^{1}$. La solution technique est un nouveau médium : elle n'implique pas de facto un nouveau langage.

Une image qui circule dans l'espace social - non pas celle qui se forme dans notre tête - est un objet matériel qui supporte une configuration visuelle, exposant une représentation de quelque ordre que ce soit $^{2}$. La partie matérielle est le médium physique qui rend possible la mise en circulation effective de l'image. La configuration visuelle est l'agencement tel qu'offert à la vue. Si les solutions d'agencement sont en nombre infini, les règles combinatoires pour produire ces solutions sont culturellement et historiquement marquées et déterminées : elle composent le langage visuel. La représentation est le résultat donné à voir de l'interprétation de quelque chose d'absent rendu présent sous quelque aspect que ce soit, suivant les spécificités du médium et les choix de configuration.

Toute discussion sur les images doit tenir compte des distinctions entre ces trois plans, médium, langage, représentation - certes indissociables puisqu'ils forment le tout qu'est l'image - au risque de confondre les enjeux cognitifs et sémiotiques avec les effets rhétoriques et esthétiques à l'œuvre. C'est à cette condition qu'il est possible de cerner comment sont engendrées les récurrences et les transformations des visions du monde exprimées par la civilisation à l'aide des images,

1 C. SAOUTER, Le langage visuel, Montréal, XYZ éditeur, 1998 (réimpressions 2000 et 2004).

2 C. SAOUTER, 2003, op. cit. 
parmi lesquelles certaines sont les empreintes d'impacts lumineux, c'est-à-dire des photographies. Sur leurs corrélations, repose la versatilité si particulière des images qui fonde leur contribution symbolique et leur poids anthropologique. Comme le dit si joliment Hans Belting, "les images sont des nomades qui campent provisoirement dans chaque nouveau médium institué au cours de leur histoire [et] ce serait une erreur de confondre images et médiums" . Dès lors, de cette distinction entre médium, langage visuel et représentation, il découle que l'ontologie photographique repose dans le plan du médium, les plans du langage et de la représentation restant ceux de tous les types d'images. Cet entendement, qui sous-tend le développement du présent exposé, détermine la discussion sur les implications d'une telle spécificité.

Cela est d'autant nécessaire et utile, d'un point de vue autant sociologique qu'épistémologique, que la photographie est devenu un moyen d'expression et de communication quasi universel.

Probablement, le meilleur facteur heuristique permettant de distinguer les phases et les aspects du corpus photographique à travers le temps, est le facteur de la diffusion, avec ce que cela implique dans un plan quantitatif, facteur qui configure le rapport de la photographie avec les autres médias, en particulier, et la culture, en général. Inhérente aux logiques des sociétés industrielles, post-industrielles et démocratiques, est donc apparue une classification sociologique distinguant une pratique amateure ${ }^{2}$, une pratique professionnelle, et une pratique artistique. Sont apparus aussi des genres, construisant une typologie d'un pôle de la documentation à un pôle de l'esthétisation, conséquente de l'ontologie photographique, dont quelques repères peuvent être : la photographie auxiliaire, le photojournalisme, le paysage, le portrait, la photographie publicitaire, la photographie de mode, la photographie d'art. Dans ces cadres, il devient possible d'inventorier un certain nombre de problèmes.

Une photographie est une configuration matérielle réalisée par empreinte - ou impact - de la lumière sur un support photosensible. Le virage numérique ne modifie pas les fondements de la photographie puisque les capteurs premiers sont toujours photosensibles et que la conversion digitale en rend compte le plus strictement possible. Ceci dit, la partie automatique de la procédure reste encadrée par un certain

1 H.BELTING, L'anthropologie des images, Paris, Gallimard, 2004, p. 273.

2 P. BOURDIEU, et al., Un art moyen, essai sur les usages sociaux de la photographie, Paris, Minuit, 1965. 
nombre de décisions prises par un sujet sémiotique, photographe amateur, professionnel ou artiste. À la prise de vue : maîtrise du flux de la lumière, choix d'une focale, d'une taille de plan, d'une profondeur de champ, option pour le noir et blanc ou la couleur, détermination d'un format horizontal, vertical ou carré, etc. Au développement et au tirage : accentuation ou atténuation des contrastes, choix d'un format, modification ou non du cadrage initial, etc. Ce que l'on a fait longtemps dans la chambre noire est fait aujourd'hui sur l'écran de l'ordinateur avec des logiciels. L'importance de ces choix, qui, d'un point de vue sémiotique, sont des interventions dans un plan dit iconique, n'invalide pas la condition d'existence sine qua non d'une configuration photographique: la captation et la conservation d'une incidence lumineuse, ce qui, d'un point de vue sémiotique, concerne un plan dit indiciaire. Quoi que l'on tente d'en penser pour y échapper, la spécificité de la photographie provient de cette double nature sémiotique indiciaire et iconique, édifiant le lieu d'une possible représentation parce que trouve place, là, une relation dialectique, vecteur d'une expression ou d'une signification possibles ${ }^{1}$.

La relation dialectique - disons, plus simplement, rhétorique entre ses deux natures, indiciaire et iconique, distingue la photographie de l'image peinte ou dessinée et de l'image de synthèse, toutes trois dans le registre seulement iconique, et qualifie le rapport de la photographie, comme chose tangible, avec ce avec quoi elle entretient une relation ontologique de ressemblance. Retenir cette caractéristique pour ordonnancer une typologie des genres photographiques, depuis un rapport d'homologie stricte jusqu'à un rapport quasi-invalidé, permet une cohérence paradigmatique et épistémologique. En effet, celle-ci autorise à construire une échelle graduée dont les intervalles sont déterminés par un même repère : le rôle ontologique de la lumière dans le fait photographique. Le rapport d'homologie le plus strict est celui qui fait de la configuration photographique une performance maximale de la fonction référentielle : c'est le domaine documentaire, celui de la photographie dite auxiliaire, dans lequel la photographie est un outil de la démarche scientifique, participant à la validité d'un passeport, d'un rapport policier, de l'ingénierie d'un pont. Le rapport quasi-invalidé se trouve dans certains types de productions artistiques, dont l'intention est d'émanciper la procédure photographique de son lien ontologique avec

1 J.-M. SCHAEFFER, L'image précaire, du dispositif photographique, Paris, Seuil, 1987. 
le référent, autrement dit, en toute logique, de réussir à faire une photographie sans utiliser la lumière. Entre ces deux extrémités, les clichés sont saisis par les débats contemporains et alimentent les questions de l'art, de l'initiative de l'artiste, de la déontologie du photoreporter, du poids socio-culturel de l'amateur.

\section{Problèmes et enjeux}

Sur la base de ces relations ontologiques et rhétoriques avec un référent, est produit depuis cent cinquante ans l'immense corpus photographique qui alimente l'Encyclopédie commune, contribue à une vision du monde, participe aux échanges symboliques. Considérant la place de la photographie dans les procès de la communication et de la culture, sont repérables quelques problématiques qui, d'une façon ou d'une autre, sous-tendent les débats actuels, scientifiques ou médiatiques.

\section{La tension entre documentation et esthétique}

Dans le continuum orienté de la pratique auxiliaire à la pratique artistique, la place médiane est occupée par le photojournalisme. Plus que tous les autres, ce genre est défini à parts égales par sa force référentielle et sa force expressive. La force référentielle donne l'indispensable valeur de vérité à ce qui est donné à voir. La force expressive provient de l'interprétation dont on accorde la légitimité au photographe. Pendant la période dite de l'âge d'or, grosso modo, des années 1930 aux années 1970, le photojournalisme a régné sans partage au sommet des virtuosités photographiques et Henri Cartier-Bresson en a expliqué les règles dans The Decisive Moment/Images à la sauvette ${ }^{1}$. Le critère prépondérant de la prise d'une bonne photographie était d'être là au bon moment et de déclencher l'obturateur à l'instant décisif. Le cliché paradigmatique de cette acception est et restera certainement celui de la mort d'un guerrier républicain pendant la guerre d'Espagne, pris par Capa en 1936, parmi nombre d'autres qui sont devenus des emblèmes du siècle. Cette production de l'âge d'or, tout en donnant ses

1 H. CARTIER-BRESSON, The Decisive Moment/Images à la sauvette, New York/ Paris, Thériade, 1952. 
quartiers de noblesse à la profession, a engendré une culture du témoignage engagé et une esthétique magnifiant le noir et blanc.

Puis est advenue l'ère de l'hégémonie télévisuelle, à laquelle se rajoutent aujourd'hui les débordements tout azimut du réseau Internet. Les tensions entre les différents médias de l'espace public, qu'on admettra ici sans les décrire ${ }^{1}$, ont poussé le photojournalisme vers, d'une part, des choix référentiels de plus en plus sélectifs, d'autre part, vers une esthétique de plus en plus expressionniste et, aujourd'hui, carrément maniériste. De l'emblème, qui relève du procès de la réception, on est passé au type, telle la figure de la victime et du déclassé, initié par le procès de la production. Sur ces types, repose un véritable académisme de la photographie sociale et de la photographie de guerre articulées plus souvent qu' autrement sur une rhétorique de la synecdoque.

Si la propension maniériste est à l'œuvre dans ce genre de photographie, dont sont exemplaires, parmi d'autres, les travaux de Sebastião Salgado, de James Nachtwey, ou encore des expositions annuelles du World Press Photo, elle trouve certainement autant d'expansions dans un genre aux antipodes : la photographie de paysages et de monuments qui assure la rentabilité de très nombreux magazines spécialisés. Ici prime l'art consommé de la métonymie qui permet d'évacuer dans le hors-champ stations services, fils électriques ou tout autre élément (mobilier, immobilier ou humain) jugés non conformes à un archétype idéal. Le rôle de ce type de clichés dans l'économie du tourisme n'est plus à faire ${ }^{2}$, mais il vaudrait certainement de scruter leur contribution dans la propagation des idéologies du patrimoine.

Le cas du photojournalisme permet clairement de cerner la tension entre documentation et esthétique, vecteur premier de la pratique photographique. En fait, toute l'histoire de la photographie peut être comprise en suivant les oscillations de cette tension, qui, à la fois, admet la capacité ontologique de la photographie à rendre compte de ce qui est et repousse la banalité présumée des basses contingences de la réalité. Les institutions françaises ont acheté les plaques de Eugène Atget sur les vues et les petits métiers de Paris mais c'est l'américaine Berenice $\mathrm{Abbot}^{3}$ puis les surréalistes qui ont fait qu'on les considère aujourd'hui non seulement comme un fonds documentaire inestimable mais aussi comme un trésor de l'art photographique. L'impact documentaire des

1 C. SAOUTER, 2003, op. cit., pp. 140-169.

2 M. PARR et S. WINCHESTER, Small World, Stockport, D. Lewis, 1995.

3 B. ABBOT, The World of Atget, New York, Horizon Press, 1964. 
clichés d'un Lewis Hine ou d'une Dorothea Lange sur l'abrogation du travail des enfants ou l'adoption du New Deal signale une fonction dans le discours délibératif : leurs images sont des arguments. En même temps, leur œuvre leur procure le statut d'artistes parce qu'ils ont construit un regard dont la puissance outrepasse les seuls enjeux pragmatiques. La Mission de la Datar (Délégation à l'aménagement du territoire et à l'action régionale) entreprend dans les années quatre vingts un recensement photographique de l'état du territoire français : elle repousse délibérément les candidats experts dans le domaine et recrute des photographes dont elle attend création libre et personnelle, tels Raymond Depardon ou Sophie Ristelhueber ${ }^{1}$. De cette dernière, toujours dans cette même perspective, on pourrait observer les circonvolutions de son travail depuis ses reportages classiques sur la guerre au Liban (1984) jusqu'à ses installations paysagères choisies pour la première guerre en Irak (1992).

\section{L'inflation quantitative et le redoublement du monde}

La production et la diffusion des images depuis la naissance de la culture dite de masse croît toujours davantage, génération technologique après génération technologique. Le phénomène est d'abord simplement mécanique. Toutes nuances faites, les avancées technologiques introduisent de nouvelles procédures ou de nouveaux médias sans complètement invalider ce qui précédait. La télévision n'a pas éliminé la photographie qui n'as pas éradiqué le dessin. Le passage au numérique n'a pas aboli l'usage du film en $35 \mathrm{~mm}$. Les cédéroms des vidéoclubs n'ont pas provoqué la disparition de l'écran de cinéma. Il s'ensuit que le phénomène est dès lors dynamique, distribuant toujours mieux les images dans les moindres recoins sociaux et culturels, en quantité toujours plus grande. Le corpus visuel contemporain est donc marqué, d'une part, par l'histoire des moyens techniques qui modèlent son apparence formelle et, d'autre part, par celle de son extension quantitative. La période concernée étant courte - à peine cent cinquante ans - ce double phénomène est perceptible par les sujets qui en sont témoins. Innovation, Révolution : ces termes du discours esthétique autant que critique ou médiatique qualifient la conscience de cette expérience. Ces termes en créent aussi une représentation qui, sociologiquement,

1 V. GuigEnO, "La France vue du sol, une histoire de La Mission Photographique de la Datar (1983-1989)", Études photographiques, no 18, 2006, pp. 97-119. 
désigne clairement une célébration faite d'ébahissement et d'enthousiasme. Sur un plan sémiotique, ces termes entretiennent et relancent le problème du rapport au monde entretenu par l'entremise des médiations visuelles.

Une première interrogation concerne le protocole de communication de toutes ces images et l'impact de leur exposition. Donner à voir des photographies fait partie intégrante du processus d'énonciation qui se termine dans le regard du récepteur. Cela est certes une évidence, si ce n'est que la compréhension des énoncés visuels est totalement dépendante d'un mode de perception en tout point identique au mode de perception du monde environnant, cause de la confusion courante entre représentation et objet de la représentation. Cela a une implication particulière sur la saisie sémiotique : alors qu'on peut suspendre le déchiffrement d'un texte, on ne peut guère suspendre le déchiffrement d'une image, photographique ou autre. La conception de la une d'un journal ou d'un magazine, d'un panneau publicitaire, d'une exposition de rue dispose de ce simple principe avec virtuosité. Cela a donné à Paris-Match sa fameuse devise, le poids des mots, le choc des photos.

Il faut alors noter le trop peu de cas fait, dans le discours réflexif, aux relations que les images entretiennent avec leur contexte d'énonciation. Objets du monde disposés à un ou des endroits du monde, se donnant à voir comme tels en même temps que donnant à voir quelque chose d'autre, les images et, plus particulièrement les photographies, offrent une multiplication de niveaux de réalité et d'interprétation. Par ailleurs, les images photographiques, réalisées grâce à un médium universel, mises en circulation dans des proportions colossales, érigent une multitude d'utilisateurs en sujets tout puissants : au ça a été du plan de l'énoncé, correspond un j'étais là du plan de l'énonciation. La prolifération des images, en l'absence de rituel d'accès, provoque dès lors ce que Marie-Josée Mondzain nomme un débordement des visibilités, faisant peser une vraie menace sur la responsabilité du regard ${ }^{1}$. Il est assez curieux de remarquer qu'il n'y a guère que la morale et la censure qui aient porté une attention précise à ce problème, longtemps avant que l'éthique laïque ne s'en saisisse, entre autres, sous la plume de Susan Sontag2.

1 M.-J. MONDZAIN, Le commerce des regards, Paris, Seuil, 2005, p. 17.

2 S. SONTAG, Regarding the Pain of Others, New York, Farrar, Straus \& Giroux, 2003 
Une seconde interrogation concerne la validation du monde par sa mise en images. A été mentionné plus haut le rôle de la photographie dans la construction des idéologies du patrimoine. Rappelons, dans un registre comparable, le rôle de la documentation photographique dans l'œuvre de Christo qui permet de rendre pérenne et commercialisable ce qui fut conçu et réalisé comme éphémère. Encore, l'intervention de Bertrand Carrière sur les plages de Dieppe, confiant à la marée les portraits photographiques des soldats tués en 1942, puis photographiant leur inéluctable destruction, matière à un livre d'artiste ${ }^{1}$. Il fut un temps où l'on convia ses familiers à d'interminables soirées de diapositives, hagiographies des dernières vacances. Aujourd'hui, les blogues personnels donnent une envergure planétaire à ce rituel. Du côté des institutions, un travail d'une ampleur sans précédent consiste, depuis au moins une décennie, à réaliser des banques d'images numériques donnant naissance, par exemple, à de véritables musées en ligne, avec tous les problèmes de reformulation des œuvres culturelles que cela soulève ${ }^{2}$. De façon symptomatique, cette propension à la documentation a donné lieu à la création, par l'UNESCO en 1997, d'une Mémoire du Monde, qui compte aujourd'hui 158 biens du patrimoine documentaire mondial.

Inscrites dans des lieux sociologiques tout à fait hétérogènes, ces pratiques ont en commun, d'un point de vue rhétorique, de promouvoir la validité d'une trace photographique, acquise grâce aux prémisses/ prémices ontologiques du ça a été. La photographie est déclarée conclusion d'un syllogisme vrai qui fait rapidement l'économie de l'intervention d'un point de vue, privé ou collectif, pourtant, dans tous les cas, interprétatif. En effet, l'attestation indiciaire de la vérité photographique autorise à passer, sans autre forme de procès, à la conviction d'un état du monde, puisque la photographie, comme objet tangible, est désormais là, dans le monde. On peut bien penser que l'amateur est un démiurge ridicule, que l'artiste est un démiurge sublime, que le scientifique est sans doute raisonnable. Ces jugements sont des lieux communs qui signalent la difficulté à penser rationnellement cette expansion quantitative d'objets porteurs de représentations en relation avec la quête des connivences autour de visions du monde.

1 B. CARRIÈRE, Dieppe, paysages et installations/Dieppe, Landscapes and Installations, Montréal, Les 400 coups, 2006.

2 C. WELGER-BARBOSA, Le patrimoine à l'ère du document numérique - Du musée numérique au musée médiathèque, Paris, L’Harmattan, 2001. 
Cette expansion quantitative et ce redoublement du monde, devant lesquels l'élite lettrée et cultivée réagit régulièrement par une tentation iconoclaste ${ }^{1}$, signalent une sorte de fuite en avant qui esquive, sinon aggrave, un problème récurrent : celui de l'inachèvement des cadres épistémologiques pour penser les formes sémiotiques issues des relations entre culture visuelle, au sens anthropologique du terme, et appréhensions du monde historiquement datées. Le chorus positiviste autour de l'avènement de l'ère numérique, ne laisse pas présager, à court terme, de progrès décisifs en la matière.

1 A. BESANÇON, L'image interdite : une histoire intellectuelle de l'iconoclasme, Paris, Fayard, 1994. 
